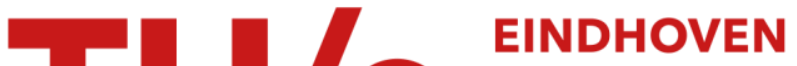 UNIVERSITY OF TECHNOLOGY
}

\section{Optical signal processing based on self-induced polarization rotation in a semiconductor optical amplifier}

\section{Citation for published version (APA):}

Calabretta, N., Liu, Y., Huijskens, F., Hill, M. T., Waardt, de, H., Khoe, G. D., \& Dorren, H. J. S. (2004). Optical signal processing based on self-induced polarization rotation in a semiconductor optical amplifier. Journal of Lightwave Technology, 22(2), 372-381. https://doi.org/10.1109/JLT.2003.822118

DOI:

10.1109/JLT.2003.822118

Document status and date:

Published: 01/01/2004

\section{Document Version:}

Publisher's PDF, also known as Version of Record (includes final page, issue and volume numbers)

\section{Please check the document version of this publication:}

- A submitted manuscript is the version of the article upon submission and before peer-review. There can be important differences between the submitted version and the official published version of record. People interested in the research are advised to contact the author for the final version of the publication, or visit the $\mathrm{DOI}$ to the publisher's website.

- The final author version and the galley proof are versions of the publication after peer review.

- The final published version features the final layout of the paper including the volume, issue and page numbers.

Link to publication

\section{General rights}

Copyright and moral rights for the publications made accessible in the public portal are retained by the authors and/or other copyright owners and it is a condition of accessing publications that users recognise and abide by the legal requirements associated with these rights.

- Users may download and print one copy of any publication from the public portal for the purpose of private study or research.

- You may not further distribute the material or use it for any profit-making activity or commercial gain

- You may freely distribute the URL identifying the publication in the public portal.

If the publication is distributed under the terms of Article $25 \mathrm{fa}$ of the Dutch Copyright Act, indicated by the "Taverne" license above, please follow below link for the End User Agreement:

www.tue.nl/taverne

Take down policy

If you believe that this document breaches copyright please contact us at:

openaccess@tue.nl

providing details and we will investigate your claim. 


\title{
Optical Signal Processing Based on Self-Induced Polarization Rotation in a Semiconductor Optical Amplifier
}

\author{
N. Calabretta, Y. Liu, Student Member, IEEE, F. M. Huijskens, M. T. Hill, Associate Member, IEEE, H. de Waardt,
} G. D. Khoe, Fellow, IEEE, and H. J. S. Dorren

\begin{abstract}
We demonstrate novel optical signal processing functions based on self-induced nonlinear polarization rotation in a semiconductor optical amplifier (SOA). Numerical and experimental results are presented, which demonstrate that a nonlinear polarization switch can be employed to achieve all-optical logic. We demonstrate an all-optical header processing system, an all-optical seed pulse generator for packet synchronization, and an all-optical arbiter that can be employed for optical buffering at a bit rate of $10 \mathrm{~Gb} / \mathrm{s}$. Experimental results indicate that optical signal processing functions based on self-polarization rotation have a higher extinction ratio and a lower power operation compared with similar functions based on self-phase modulation.
\end{abstract}

Index Terms-Nonlinear polarization switch (NPS), optical arbiter, optical buffering, optical header processor, optical self-synchronization, optical signal processing, self-induced effects, semiconductor optical amplifier (SOA).

\section{INTRODUCTION}

$\mathbf{S}$ ELF-INDUCED effects in nonlinear optical mediums can be employed to realize all-optical signal processing functions. These effects occur when an optical signal introduces a change in a nonlinear medium through which the signal propagates while the medium reacts back on the signal itself. Semiconductor optical amplifiers (SOAs) have been employed as a nonlinear element in all-optical logic gates. An example of a self-induced optical effect in an SOA is self-phase modulation. This effect has been employed to realize optical header processing [1]-[3], optical threshold functions [4], [5], optical self-synchronization [6]-[9], and optical clock recovery [10]. Most of these optical signal processing functions have been realized by employing self-induced optical effects in combination with an interferometer.

Recently, considerable attention has been paid to optical signal processing based on nonlinear polarization switches (NPSs) [11], [12]. Examples include wavelength conversion [13] and all-optical flip-flop memories [14]. A model for a nonlinear polarization rotation switch has been presented in [15]. The examples mentioned previously are based on nonlinear polarization rotation in an SOA in which the polarization state of a probe pulse is controlled by external (saturating) pump

Manuscript received March 19, 2003; revised September 22, 2003. This research was supported by the Netherlands Organization for Scientific Research (NWO) under "NRC Photonics" and "VIDI" grants.

The authors are with the Cobra Research Institute, Eindhoven University of Technology, 5600 MB Eindhoven, The Netherlands.

Digital Object Identifier 10.1109/JLT.2003.822118

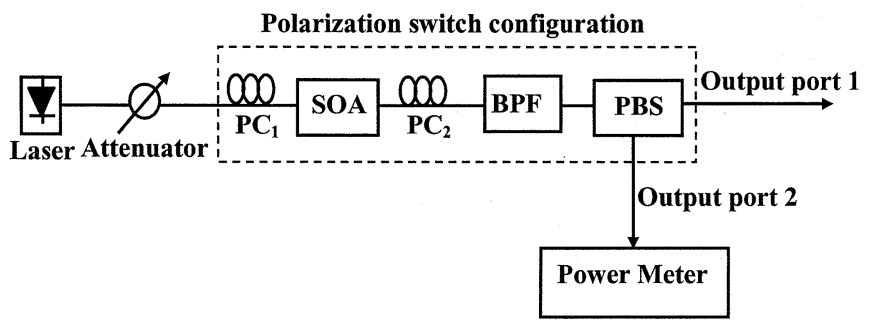

Fig. 1. Experimental setup that is used to measure self-polarization rotation in an SOA. PC: Polarization controller; SOA: semiconductor optical amplifier; BPF: bandpass filter; PBS: polarization beam splitter.

light. We believe that up until now, no results are published in which the NPS is driven by self-induced effects.

In this paper, we investigate self-induced polarization rotation (SPR) in an SOA in the context of all-optical signal processing. We will show that the polarization-dependent gain saturation model presented in [15] can be applied to describe SPR in an SOA. In the case of SPR, the role of the saturating control beam is taken over by the data bits themselves. We will also show that SPR in an SOA in combination with a NPS can be employed for all-optical signal processing. In order to illustrate the potential of this concept for telecommunication technology, we present an all-optical header processor, an all-optical seed-pulse generator, and an optical arbiter based on SPR.

The paper is organized as follows. In Section II, we demonstrate SPR in an SOA and a NPS based on SPR. In Section III, applications of all-optical signal processing based on SPR are presented. We demonstrate an all-optical header processing system, an optical seed-pulse generator for packet synchronization and an all-optical arbiter that could be employed in a packet buffer at a data rate of $10 \mathrm{~Gb} / \mathrm{s}$. The paper is concluded with a discussion.

\section{SElf-Polarization Rotation}

The general configuration of a NPS is depicted in the dashed box in Fig. 1. In [15], a model for nonlinear polarization rotation driven by polarization-dependent gain saturation in an SOA is presented. In brief, the polarization switch operates in a similar fashion to a Mach-Zehnder interferometer (MZI) switch, but the role of the different arms is taken over by the transverse electric (TE) and transverse magnetic (TM) modes of the incoming light. The modes propagate independently through the SOA, but they have indirect interaction via the carriers. In the model presented in [15], the TE and TM modes couple to two 
different reservoirs of holes. Thus, if the SOA is saturated by an optical control signal, the gain saturation of the TE mode differs from the gain saturation of the TM mode [15]. Hence, the refractive index change of the TE mode also differs from the refractive index change of the TM mode. If a small probe signal with well-defined polarization is simultaneously injected into the SOA with a saturating pump signal, a phase difference between the modes builds up as the light propagates through the SOA. When the two modes recombine at the polarization beam splitter (PBS), the phase difference determines to which of the output ports of the PBS the signal is switched [15]. We use in our experiments a commercially available strained-bulk SOA (produced by JDS-Uniphase). This device is similar to the one that is used in [15], and thus the modeling results presented in that reference can be used to describe the system applications that follow. In [15], the presence of tensile strain in the SOA was modeled by using a population imbalance factor $f$ that has to be determined from characterization measurements. The model presented in [15] can also be used to model nonlinear polarization rotation in unstrained SOAs by putting $f=1$. In the latter case, the nonlinear polarization rotation is described by the different confinement factors of the TE and TM modes, and thus for $f=1$, the model in [15] is equivalent to that presented in [16].

In the case of SPR, the phase difference between the TE and TM modes is created by the signal itself. When an optical bit with sufficient optical power arrives at the SOA, the leading edge of the bit introduces gain saturation in the SOA. Since the SOA gain saturation is polarization dependent, the TE component of the data bit experiences different gain saturation than the TM component. Thus, the leading edge of a data bit can introduce a rotation of the polarization state.

In the following experiment, we demonstrate that an optical switch can be driven by SPR. The experimental setup that is used to demonstrate the SPR concepts is given in Fig. 1. The laser source emits continuous-wave $(\mathrm{CW})$ light at a wavelength of $1550.91 \mathrm{~nm}$ that is injected into the polarization switch via an optical attenuator. The polarization switch consists of an SOA, two polarization controllers (PCs), and a PBS. At the PBS, both modes recombine. $\mathrm{PC}_{1}$ is used to adjust the polarization of the input signal with respect to the SOA layers (the angle between the TE and TM modes is approximately $45^{\circ}$ [14]). $\mathrm{PC}_{2}$ is used to adjust the polarization of the SOA output with respect to the orientation of the PBS. As an initial experiment, to characterize the population imbalance factor $f$, we measure the output power at output port 2 as a function of the pump current. The attenuator was set in such a way that the power of the CW light that enters the $\mathrm{SOA}$ via $\mathrm{PC}_{1}$ was equal to $-20 \mathrm{dBm}$. This guarantees that the $\mathrm{CW}$ light does not saturate the SOA. $\mathrm{PC}_{2}$ was set in such a way that no light outputs at port 2 . The solid line in Fig. 2 shows the power at output port 2 as a function of the SOA pump current. It can be observed that the power of output port 2 increases as the pump current increases. The population imbalance factor $f$ can be determined as a function of the SOA injection current from the curve presented Fig. 2 by following a similar approach as used in [15]. We find that $f$ varies in a range of 0.5 and 0.6 if the SOA injection current varies between $70 \mathrm{~mA}$ and $390 \mathrm{~mA}$. All the other parameters that are used in modeling

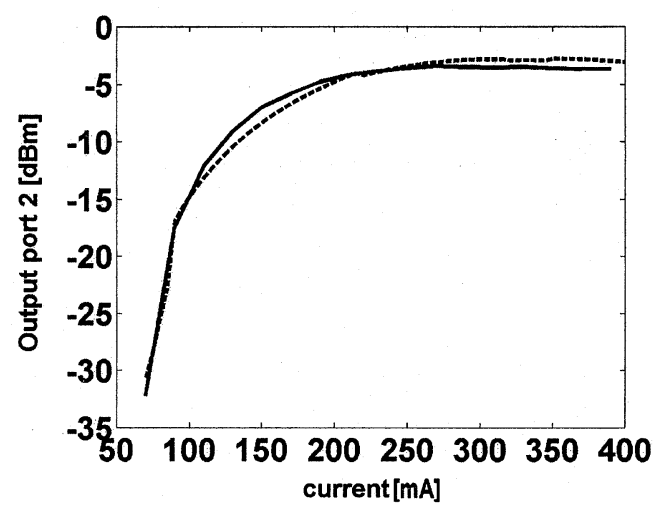

Fig. 2. Curves showing the measured optical power (solid line) and computed optical power (dashed line) at output port 2 as a function of the SOA pump current.

TABLE I

Parameters USEd in the Simulations of SElF-Polarization ROTATION IN AN SOA

\begin{tabular}{|c|}
\hline$T=500 p s$ \\
\hline$f=0.52$ \\
\hline$\kappa=110^{-3} m A^{-1}$ \\
\hline$N_{0}=10^{8}$ \\
\hline$v_{g}^{T E}=v_{g}^{T M}=100 \mu \mathrm{m} / \mathrm{ps}$ \\
\hline$\Gamma^{T E}=0.2$ \\
\hline$\Gamma^{T M}=0.14$ \\
\hline$\xi^{T E}=710^{-9} \mathrm{ps}^{-1}$ \\
\hline$\xi^{T M}=6.510^{-9} \mathrm{ps}^{-1}$ \\
\hline$e=1.610^{-19} \mathrm{C}$ \\
\hline$\alpha_{i n t}^{T E}=\alpha_{i n t}^{T M}=0.27 p^{-1}$ \\
\hline$\alpha_{T E}=\alpha_{T M}=5$ \\
\hline$L=800 \mu \mathrm{m}$ \\
\hline$h=6.62610^{-34} \mathrm{Js}$ \\
\hline
\end{tabular}

results throughout this paper are listed in Table I. The dashed curve in Fig. 2 shows the computed output power as a function of the injection current. This curve is computed by using the model of [15]. It can be observed that the simulations are in excellent agreement with the measurements.

In the second experiment, we measure the static behavior of the polarization switch as a function of the power of the input light. In this case, the SOA current is set to $170 \mathrm{~mA}$, and $\mathrm{PC}_{2}$ was set in such a way that the light is suppressed at output port 2 for low optical input power. The optical power at output port 2 


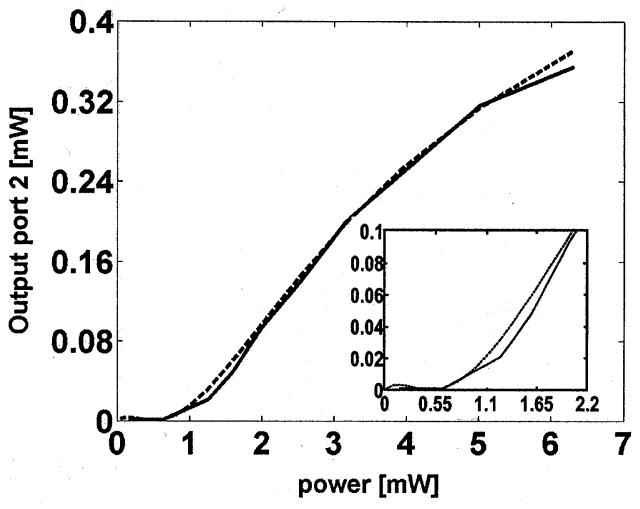

Fig. 3. Curves showing the measured optical power (solid line) and computed optical power (dashed line) at output port 2 as a function of the intensity of the $\mathrm{CW}$ input optical power. The inset represents a zoom in the switching region.

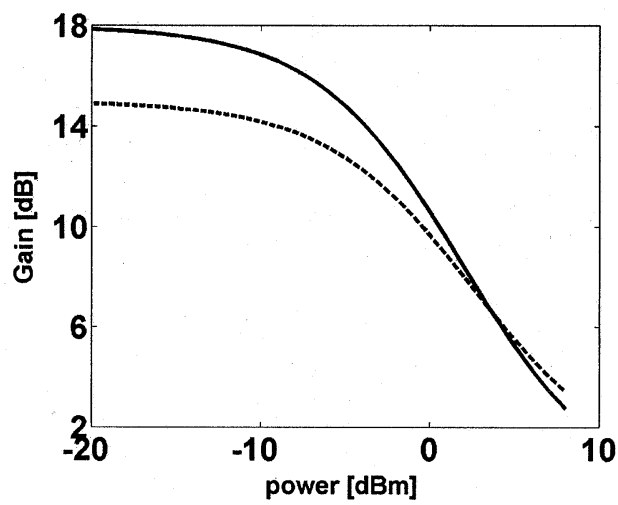

Fig. 4. Computed SOA gain for the TE (solid line) and the TM (dashed line) modes as a function of the intensity of the input signal.

has been measured as a function of the power of the input light. The result is shown by the solid line in Fig. 3, from which it can be observed that the output power at port 2 increases as a function of the input power. The shape of the curve in Fig. 3 matches with the theoretical model of [15] which predicts that the relationship between the output power at port 2 and the polarization angle $(\Delta \theta)$ is proportional to $1-\cos (\Delta \theta)$. The dashed line in Fig. 3 represents a simulation result for the output power of the NPS using the model of [15]. In the simulations, $f$ is set to 0.52 , and moreover, the optical input power used in the computations has been increased by $4.6 \mathrm{~dB}$ to compensate for the insertion losses $\left(1.3 \mathrm{~dB}\right.$ for each $\mathrm{PC}_{1}$, and $3.3 \mathrm{~dB}$ for the facet losses, including the connector losses). It is clearly visible in Fig. 3 that the modeling results are in excellent agreement with the measured data. In Fig. 4, the computed gain for the TE and TM modes is presented. It can be observed that the difference between the TE gain (solid line) and the TM gain (dashed line) decreases when the input power increases. The gain difference introduces a phase difference between the modes that results in a rotation of the polarization angle [15]. Since the output power at port 2 changes as a function of the polarization angle $(\Delta \theta)$ according to $1-\cos (\Delta \theta)$, for small $\Delta \theta$, the transfer function can be approximated by $\Delta \theta^{2}$. This indicates that a linear change in the input power leads to a quadratic change in the polarization rotation and thus a large variation in the output power. We measured a static contrast ratio larger than $18 \mathrm{~dB}$ between the output light in the case that $\Delta \theta=0$ (intensity of the input light was $0.63 \mathrm{~mW}$ ) and the output light in the case that $\Delta \theta$ has been changed by increasing the intensity of the input light of $3 \mathrm{~dB}$ with respect to the case that $\Delta \theta=0$ (see also the inset of Fig. 3). In addition, the dynamic contrast ratio has been measured for a nonreturn-to-zero (NRZ) data signal at a bit rate of $2.5 \mathrm{~Gb} / \mathrm{s}$. The experimental setup is shown in Fig. 5. In Fig. 6(a) and (b), the input signals with average powers of $0.63 \mathrm{~mW}$ (corresponding to $\Delta \theta=0)$ and $1.35 \mathrm{~mW}$ are presented. In Fig. $6(\mathrm{c})$ and (d), the traces measured at port 2 are presented. It follows that a signal with an average power of $0.63 \mathrm{~mW}$ cannot pass through the PBS [see Fig. 6(c)], while the signal with higher input power (1.35 $\mathrm{mW}$ ) can pass through the PBS [see Fig. 6(d)]. The dynamic contrast ratio was measured larger than $10 \mathrm{~dB}$.

These results provide evidence that a NPS can be driven by SPR in an SOA and also that the model presented in [15] can explain SPR in an SOA. In the next section, we will demonstrate applications of all-optical signal processing functionalities based on NPS, which can be applied in all-optical packetswitched cross-connects.

\section{Applications to All-Optical Logic}

An optical packet-switched cross-connect that uses all-optical signal processing to route data packets is presented in [17]. This paper illustrates that three main functions have to be realized in order to achieve all-optical packet switching: all-optical synchronization, all-optical buffering, and all-optical packet switching.

In the following sections, we present all-optical signal processing functions based on SPR that can be employed for all-optical header processing. We also present an all-optical seed-pulse generator for packet synchronization, and finally, we present an all-optical arbiter that can be employed for packet buffering.

\section{A. All-Optical Header Processor Based on SPR}

An all-optical correlator is a fundamental building block to realize all-optical header recognition [1]-[3], [17]. We present an optical correlator based on the NPS that is discussed in the previous section. We also present an application of this optical correlator for all-optical header recognition.

The optical correlator is presented schematically in the dashed box of Fig. 7. The optical power of the data signal is split by a coupler into one data signal that enters the SOA on the right side (to be called henceforth the left propagating data signal) and one data signal that enters the SOA (via the optical circulator) on the left side (to be called henceforth the right propagating data signal). The right propagating data signal is first delayed by a time $\tau_{L}$ and then fed into the SOA of the optical correlator via an optical circulator. $\mathrm{PC}_{2}$ is set to switch the light to the output port 1 , only if the left propagating data signal passes through the SOA. When the right propagating data signal also enters the SOA, one can distinguish two cases. In the first case, the optical power of the right propagating data signal is not sufficient to saturate the SOA. As a result, the left propagating data signal experiences no polarization rotation. Thus, the left propagating data signal is switched to output port 


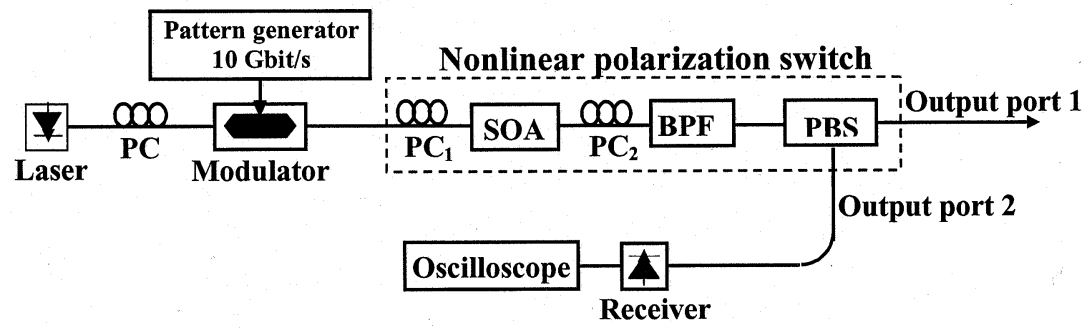

Fig. 5. Experimental setup of the NPS. PC: Polarization controller; SOA: semiconductor optical amplifier; BPF: bandpass filter; PBS: polarization beam splitter.
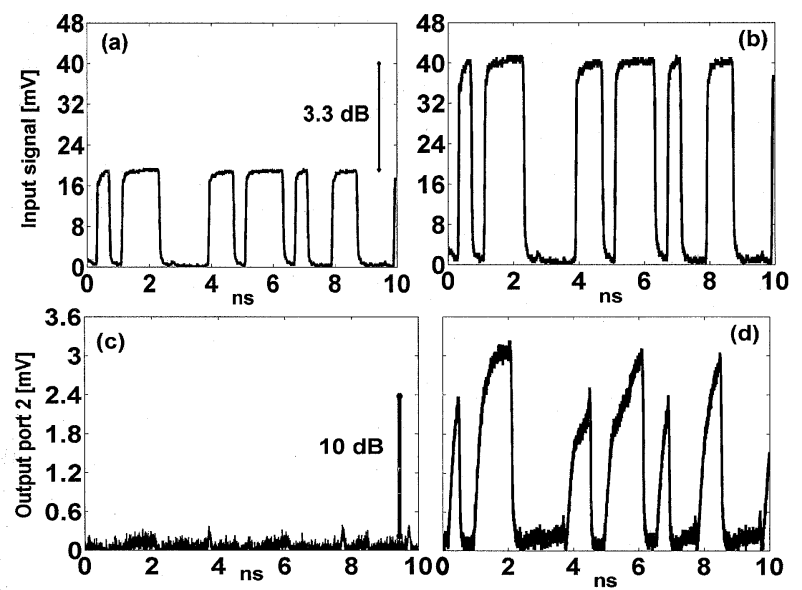

Fig. 6. Experimental switching results for the NPS. (a) Optical input signa (average power 0.63). (b) Optical input signal (average power 1.35). (c) Trace of output port 2 for an input signal with average optical power of $0.63 \mathrm{~mW}$ (d) Trace of output port 2 for an input signal with average optical power of 1.35 $\mathrm{mW}$.

1. In the second case, the optical power of the right propagating data signal is sufficient to saturate the SOA. In this case a correlation between the left propagating and the delayed right propagating data signal is formed since the left propagating data signal experiences a saturated SOA and thus polarization rotation. As a result, the left propagating data signal is switched to output port 2. Moreover, the time window in which pulse correlation can take place is $\tau_{e}$, where $\tau_{e}$ is the time that the SOA needs to recover from the saturation state.

The optical correlator can function as an all-optical header processor as follows. The output port 2 represents the output of the header processor. Suppose that the header addresses consist of two header pulses and that the displacement (in time) between two header pulses is $\tau$ (see packet format in Fig. 7). A header correlation pulse is formed at the output port 2 if $\tau_{L}-\tau<\tau_{e}$, since the second left propagating header pulse experiences a saturated SOA introduced by the first right propagating header pulse, while no header correlation pulse is formed at the output port 2 if $\tau-\tau_{L}>\tau_{e}$ or $\tau_{L}-\tau>\tau_{e}$ since in this case, all pulses propagate through an unsaturated SOA [2].

The header processing system is schematically presented in Fig. 7. It consists of a header preprocessor (HPP) [3] followed by the header processor discussed previously. The optical packet format is also presented in Fig. 7. The header section consists of two header pulses that are separated by a sequence of alternating NRZ "0" and " 1 " bits at the same bit rate as the data payload, with the exception of a sequence of "0"s with duration longer than the recovery time of the SOA which is placed in front of

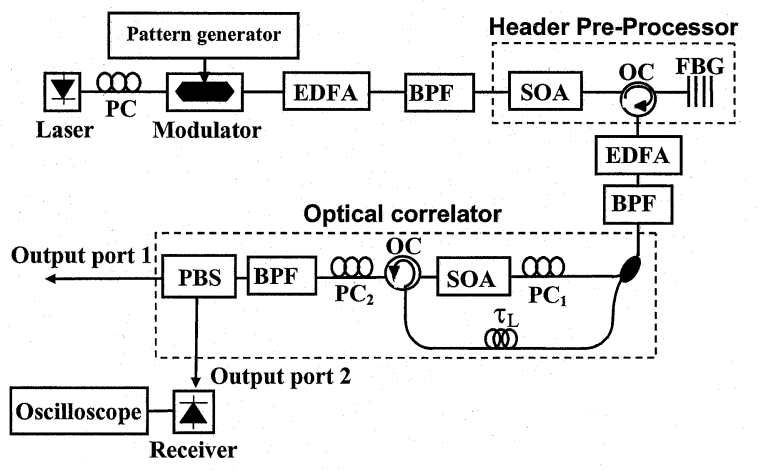

Packet format:

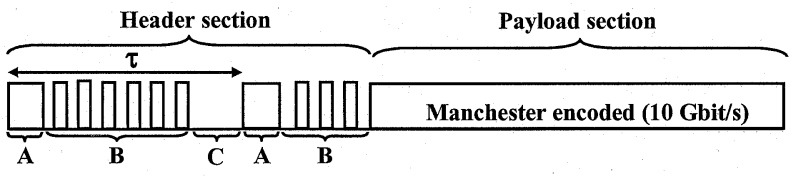

A - Two NRZ header pulses $(2.5 \mathrm{Gbit} / \mathrm{s})$

B - Alternates ' 1 ' and ' 0 ' bits (10Gbit/s)

C - Sequence of NRZ '0s' (2.5 Gbit/s)

Fig. 7. Experimental setup of the header processing system. The packet format of the optical packets employed in the experiments is also shown. EDFA: Erbium-doped fiber amplifier; BPF: bandpass filter; PC: polarization controller; SOA: semiconductor optical amplifier; OC: optical circulator; FBG: fiber Bragg grating; $\tau_{L}$ : delay of the counterpropagating signal; PBS: polarization beam splitter.

the second header pulse. The position of the second header pulse within the header section is used to define a unique header pattern. Moreover, the space between the second header pulse and the payload is also filled with the sequence of alternating NRZ "0" and " 1 " bits. The sequence of alternating NRZ " 0 " and " 1 " bits is used to keep the SOA in saturation when the packet passes through the SOA, while the sequence of " 0 "s gives time to the SOA to recover before the second header pulse passes through the SOA. The payload was Manchester encoded to avoid repetition of the header pattern in the packet payload. Manchester encoding also guarantees that the average signal power of the payload is constant, regardless of the specific bit pattern.

As an example, we assume packets with only two different header patterns. The first packet has a header section consisting of a hexadecimal "FAAAAAA00FA" pattern (Header 1). For this header pattern, the time between the header pulses is $\tau_{1}$ [see Fig. 8(a)]. The second packet has a header section consisting of a hexadecimal "FAAAAAAA00F" pattern (Header 2) corresponding to a time $\tau_{2}$. First, the packets are preprocessed by an HPP that separates the two header pulses from the packet 
payload. The operation of the HPP based on self-phase modulation (SPM) in an SOA is described in [3]. The output signal of the HPP consists of the leading edges of the two NRZ header pulses while the rest of the packet is suppressed [see Fig. 8(b)]. The output of the HPP is fed into the optical-correlator-based header processor. We choose the delay $\tau_{L}$ in such a way that $\tau_{L}-\tau_{1}<\tau_{e}$ and $\tau_{2}-\tau_{L}>\tau_{e}$. Thus, only a correlation pulse is formed at output port 2 for packets with Header 1 (since $\tau_{L}-\tau_{1}<\tau_{e}$ ). No correlation pulse is formed at the output port 2 for packets with Header 2 since $\tau_{2}-\tau_{L}>\tau_{e}$.

The concepts described previously were demonstrated by using the experimental setup shown in Fig. 7. The laser source had a wavelength of $1558.34 \mathrm{~nm}$ and was modulated by a 10-Gb/s Mach-Zehnder modulator (MZM), which was driven by a pulse-pattern generator. The packet format is shown in Fig. 7. The packets had a header section that consists of two NRZ header pulses at a bit rate of effectively $2.5 \mathrm{~Gb} / \mathrm{s}$. The header pulses are separated by a sequence of alternating NRZ " 0 " and " 1 " bits at the same bit rate of the packet payload. The duration of the sequence of " 0 "s that were placed in front of the second header pulse was to $0.8 \mathrm{~ns}$. The payload section consisted of a Manchester-encoded pseudorandom bit sequence (PRBS) data at a bit rate of $10 \mathrm{~Gb} / \mathrm{s}$. The SOA in the HPP was pumped with $204 \mathrm{~mA}$ of current. The fiber Bragg grating (FBG) employed had a reflectivity of $99.9 \%$ at $\lambda=1558.93 \mathrm{~nm}$ with a bandwidth equal to $0.43 \mathrm{~nm}$.

The optical packets used in the experiment consisted of sequential optical packets with Header 1 and Header 2 as shown in Fig. 8(a). The time between the leading edges of the two header pulses was equal to $\tau_{1}=3.8 \mathrm{~ns}$ and $\tau_{2}=4.8 \mathrm{~ns}$ for the Header 1 and Header 2, respectively. The average optical power of the packets at the input of the HPP was $4 \mathrm{dBm}$. The oscilloscope trace of the signal at the output of the HPP is presented in Fig. 8(b). It is clearly visible in Fig. 8(b) that the HPP generates a pulse at the leading edges of the header bits. The average payload suppression is $11.4 \mathrm{~dB}$. The output of the HPP is then amplified by an erbium-doped fiber amplifier (EDFA), filtered by a 2-nm bandpass filter and fed into the header processor (see Fig. 7). The average optical power of the left and right propagating signals was -8 and $2.35 \mathrm{dBm}$, respectively (before the optical circulator). The SOA in the header processor was pumped with $178 \mathrm{~mA}$ of current. The PBS has an extinction ratio of $30 \mathrm{~dB}$. The delay was $\tau_{L}=3.8 \mathrm{~ns}$ that matches the time $\tau_{1}$ between the leading edges of pulses in Header 1 . Thus, we expect a correlation pulse only for optical packets with Header 1. This is confirmed in Fig. 8(c), where only for packets with Header 1 a correlation pulse is formed. The contrast ratio between the average optical power of the header correlation pulse and the suppressed payload is equal to $18.6 \mathrm{~dB}$. This is an improvement of $4.4 \mathrm{~dB}$ compared with the results presented in [1], [2], [17].

The experimental results indicate that the header processing system can distinguish between two different header patterns. A limitation of this approach is that that a packet header only contains two bits of information. The length of the packet header can be extended by creating larger packet headers that are built up out of combinations of two bits (in a similar fashion as in [2]). The minimum interval between different header bits is de-
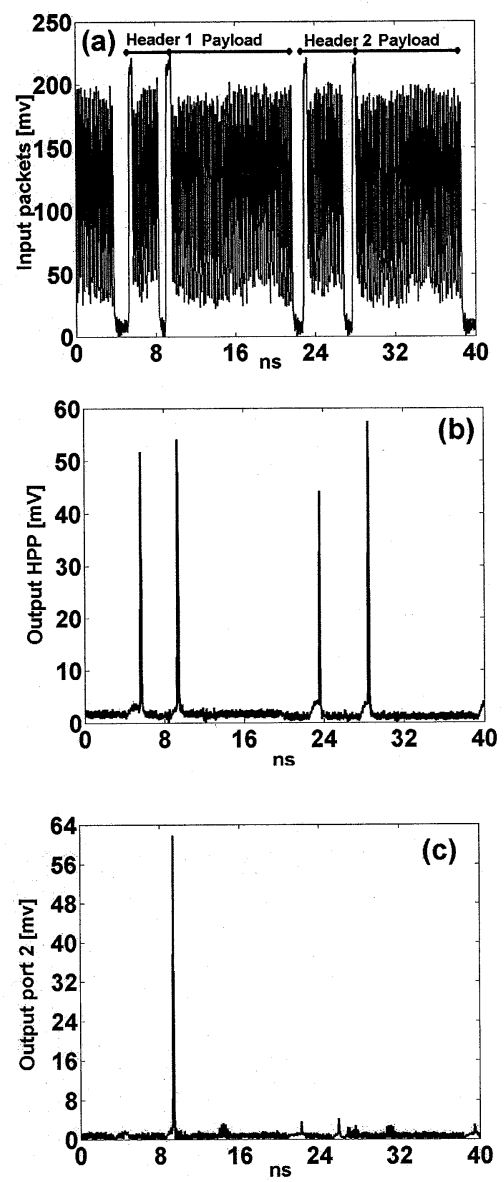

Fig. 8. Experimental results demonstrating the operation of the header processor. (a) Optical input signal. (b) Output of a header preprocessor based on SPM. (c) Output of header processor (measured at the output port 2).

termined by the SOA recovery time ( $\sim 1 \mathrm{~ns})$. This means that the duration of the packet is proportional with the number of header bits times the SOA recovery time. It should be noted, however, that the SOA recovery time can be decreased to $60 \mathrm{ps}$ [18]. This means that the length of header patterns can be reduced with more than a factor 10 .

\section{B. All-Optical Header Preprocessor Based on SPR}

We will show that the NPS of Fig. 5 can also be used as a header preprocessor. In this case, we set $\mathrm{PC}_{2}$ in such a way that the unsaturated input data packets are switched to port 2.

When a data pulse enters the NPS, the leading edge will saturate the SOA. In Section II, it is explained that the SOA gain saturation is polarization dependent, and hence, rotation of the polarization angle takes place. This ensures that only the leading edge of a data pulse is switched to output port 2 (for the leading edge the SOA is not yet saturated), while the rest of the bit leaves the NPS at port 1 (since the SOA is saturated and the polarization angle of the light is changed).

When a packet with a header as described in the previous subsection enters the header preprocessor, the leading edges of both header pulses are routed to port 2 . The packet payload and the remainder of the header pulse are switched to port 1.

Assume that the optical packets have a packet format as shown in Fig. 7. The sequence of NRZ "0"s placed in front 

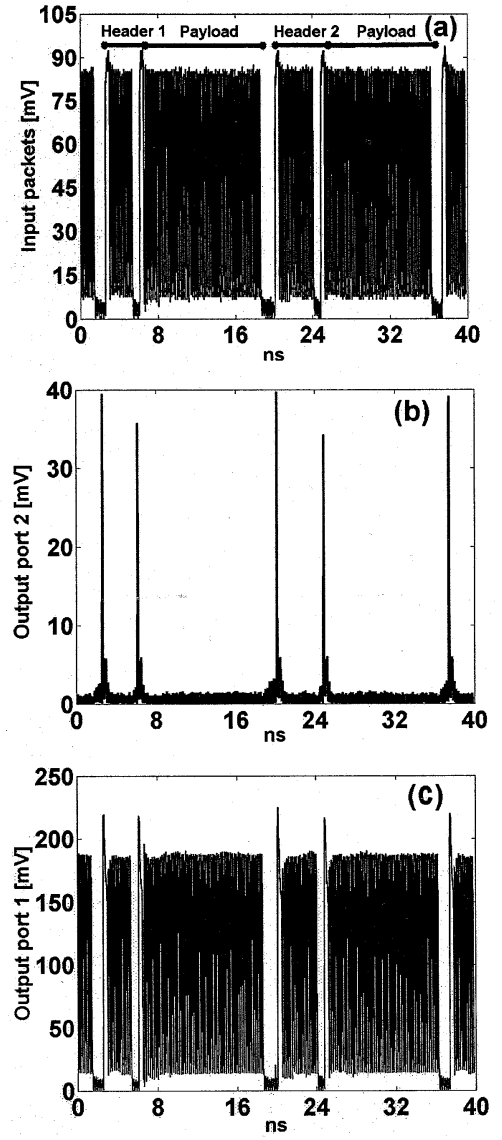

Fig. 9. Experimental results of the header preprocessor based on NPS. (a) Optical input signal. (b) Output of the header preprocessor (measured at the output port 2). (c) Amplified copy of the input signal (measured at the output port 1).

of the NRZ header pulse is longer than the gain recovery time of the SOA. The transitions of the Manchester-encoded data payload and the alternating " 1 " and " 0 " bits in the header section are faster than the gain recovery time of the SOA. This packet format guarantees that when the optical packet enters the header preprocessor, only the leading edges of the two NRZ header pulses experience full gain, while the rest of the packet experiences the SOA in saturation. Thus, the leading edges of the two header pulses are switched to output port 2 , and the rest of the packet is switched to output port 1 .

The experimental setup used to demonstrate the header preprocessor is shown in Fig. 5. The laser source had a wavelength of $1550.91 \mathrm{~nm}$ and was modulated by a 10-Gb/s MZM, which was driven by a pulse-pattern generator. The packets employed in the experiments are shown in Fig. 9(a). The SOA was pumped with $141.5 \mathrm{~mA}$ of current. The average optical power of the packets measured before the SOA was $-0.2 \mathrm{dBm}$. The measured output signals at output port 1 and output port 2 are shown in Fig. 9(b) and (c). Output port 2 represents the output of the header preprocessor. The extinction ratio between the header pulses and the suppressed payload is larger than $15 \mathrm{dBm}$, which is an improvement of circa $4 \mathrm{~dB}$ with respect to the HPP presented in [3]. In addition, output port 1 provides an amplified copy of the input packets. Moreover, the header preprocessor operates at much lower power compared with the HPP presented in [3].
In conclusion, we have demonstrated an alternative header preprocessor based on an NPS that can operate at a bit rate of 10 $\mathrm{Gb} / \mathrm{s}$. Higher operation speed is possible by decreasing the recovery time of the SOA [18]. This header preprocessor presents several advantages over HPP: lower power operation, higher extinction ratio, and an extra output port that provides an amplified copy of the packet.

\section{All-Optical Seed-Pulse Generator Based on SPR}

An optical packet-switched cross-connect requires bit-level synchronization and phase alignment for each packet in order to perform signal processing and routing [7]. Self-synchronization is one of the methods employed to achieve bit-level synchronization [7]-[9]. In general, a self-synchronization method requires a seed-pulse synchronized at the beginning of the packet. The pulse can be used to generate a local clock for packet synchronization [6]-[9], [20]. An advantage of the self-synchronization approach is that it is insensitive to timing jitter between packets or network architecture [19].

We present an all-optical seed-pulse generator based on the NPS described in the previous section. The optical seed-pulse generator is schematically shown in Fig. 10. It consists of an asymmetric passive MZI followed by the NPS. The format of the packets that are used is shown in Fig. 7. The role of the MZI is to merge the packet with a delayed copy of itself. It is essential that the delay is shorter than the interval between the header bits. As a result, at the MZI output, the specific packet structure is lost. When the MZI output enters the NPS, only the leading edge of the first NRZ header pulse experiences the unsaturated gain of the SOA, while the remainder of the data packet experiences a saturated gain. Thus, only the leading edge of the first NRZ header pulse is switched to output port 2 , while the remainder of the data packet is switched to output port 1. The pulse switched to output port 2 is synchronized with the beginning of the packet, and this represents the seed pulse.

The experimental setup used to demonstrate the optical seedpulse generator is shown in Fig. 10. The laser source had a wavelength of $1550.91 \mathrm{~nm}$ and was modulated by a $10-\mathrm{Gb} / \mathrm{s}$ MZM, which was driven by a pulse-pattern generator. The input packets employed in the experiments are shown in Fig. 11(a). The asymmetry $\tau_{d}$ between the two arms of the MZI was measured to be $\sim 1 \mathrm{~ns}$. The SOA was pumped with $142.5 \mathrm{~mA}$ of current. The average optical power of the packets measured before the MZI was $1.5 \mathrm{dBm}$.

In the first experiment, we demonstrate that the optical seedpulse generator forms a seed pulse synchronized with the beginning of the packet. The output of the optical seed-pulse generator is shown in Fig. 11(b). The seed pulses, synchronized with the beginning of the packets, are clearly visible. The extinction ratio between the seed pulse and the suppressed packet is larger than $15 \mathrm{~dB}$.

In the second experiment, we show that the optical seed-pulse generator can still extract timing information, even when the input data packet has an extinction ratio of $5 \mathrm{~dB}$. This confirms that the optical seed-pulse generator can be employed as a first stage to generate a local clock for synchronization purposes. The optical input packet is shown in Fig. 12(a). The output of the optical seed-pulse generator is presented in Fig. 12(b). The 


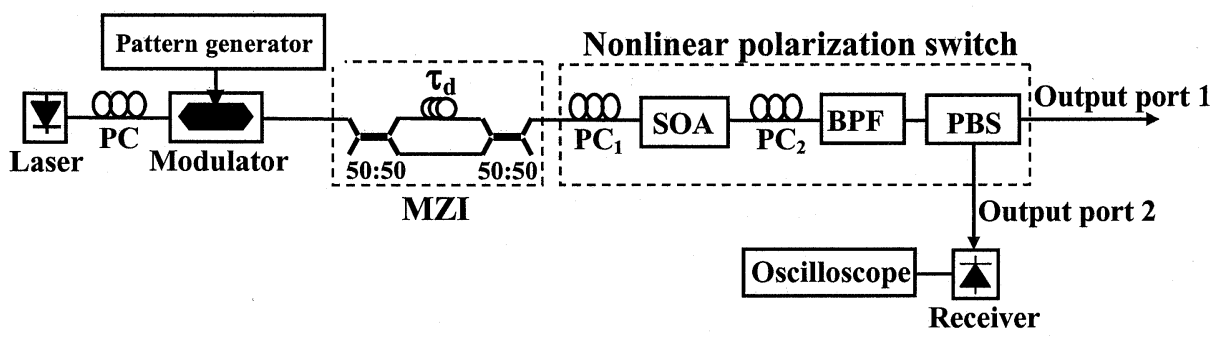

Fig. 10. Experimental setup of the optical seed-pulse generator. MZI: Asymmetric Mach-Zehnder interferometer; $\tau_{d}$ : time delay between the two arms of the MZI.
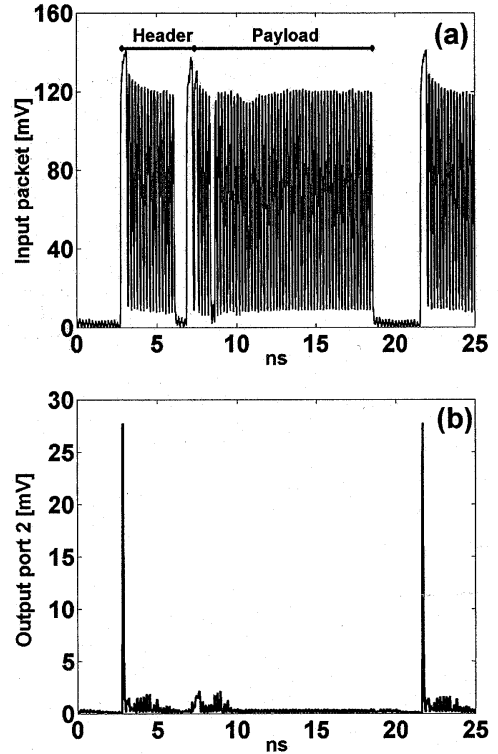

Fig. 11. Experimental results demonstrating the operation of the optical seed-pulse generator. (a) Optical input signal. (b) Output of the self-synchronizer (measured at the output port 2).

seed pulses synchronized with the beginning of the packets are clearly visible in Fig. 12(b). The measured extinction ratio between the average power of the seed pulse and the suppressed payload is over $10 \mathrm{~dB}$. However, since we set $\mathrm{PC}_{2}$ in such a way that the input data are switched to port 2 , the power of the guard time between the packets also leaves port 2 . This leads to a reduction of the extinction ratio with $5 \mathrm{~dB}$. The extinction ratio can be increased by cascading another NPS or an optical threshold function similar to that presented in [1], [5] after the optical seed-pulse generator.

\section{All-Optical Arbiter Function}

The header processing system demonstrated in Section III-A can only handle one packet at any given moment. Therefore, optical buffers are necessary to avoid packet contention. In [17] and [21], an all-optical buffering concept is demonstrated based on a wavelength routing approach. The arbiter function was obtained by using an optical threshold function. The functionality of the optical threshold function is twofold: it acts as an arbiter to decide whether packet contention takes place, and it also controls a wavelength routing switch. The wavelength routing switch consists of a wavelength converter followed by a demultiplexer.

We present a novel all-optical arbiter function (OAF) based on an NPS that can indicate whether packet contention takes
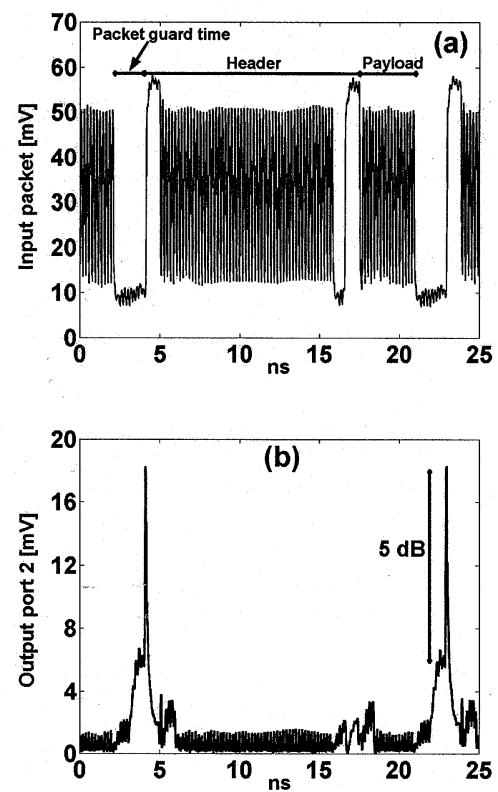

Fig. 12. Experimental results demonstrating the operation of the optical seed-pulse generator. The extinction ratio of the input data packet employed in this experiment is $5 \mathrm{~dB}$. (a) Optical input signal. (b) Output of the self-synchronizer (measured at the output port 2).

places. The OAF consists of a MZI and a NPS as schematically shown in Fig. 13. The packet format is shown in Fig. 7. We distinguish between a packet with low priority (packet 1 ) and a packet with high priority (packet 2). Packet 1 enters the NPS via the MZI, while packet 2 enters the NPS via the optical circulator (OC). We assume that the packets arrive synchronously at the NPS. If no contention takes place, only packet 1 enters the NPS. In that case the NPS acts as the self-synchronizer discussed before. Thus, a single output pulse corresponding to the leading edges of the NRZ header pulse is formed at output port 2. When packet contention takes place, packet 1 and packet 2 arrive simultaneously at the NPS. In this case packet 1 experiences a saturated SOA, introduced by packet 2 and thus the polarization angle of packet 1 is rotated. Hence, no output pulse is formed at output port 2. The OAF can be employed in a buffering system as follows [17], [21]. The pulse at the output of the OAF (output port 2) can be used to set/reset a flip-flop memory [1], [5], [17]. The output of the flip-flop controls an optical wavelength routing switch.

The experimental set-up used to demonstrate the concept of the OAF is shown in Fig. 13. The laser source had a wavelength of $1550.91 \mathrm{~nm}$ and was modulated by a $10 \mathrm{~Gb} / \mathrm{s}$ Mach-Zehnder modulator, which was driven by a pulse-pattern generator. The 


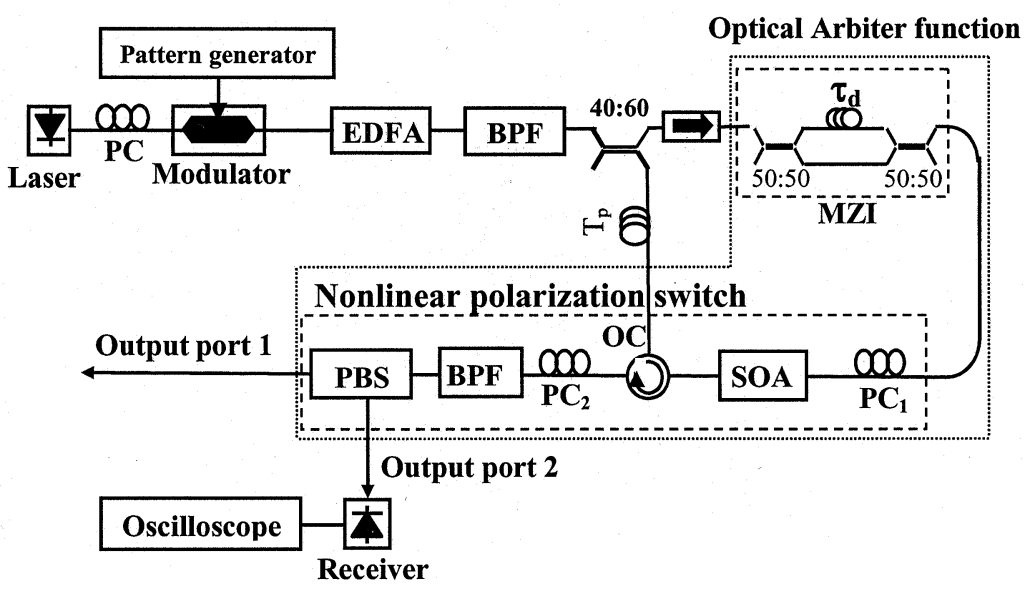

Fig. 13. Experimental setup for the all-optical arbiter. $T_{p}$ : time delay equal to one packet slot.

packets employed in the experiment are shown in Fig. 14(a). Two sequential optical packets were used in the experiments. Packet 1 contains a header pattern consisting of a hexadecimal "FAAAAAA00FA" bit pattern. Packet 2 contains a header pattern consisting of a hexadecimal "FAAAAAAA00F" bit pattern. Also visible in Fig. 14(a) is a third dummy packet that consists of a sequence of " $0 \mathrm{~s}$ " with a length equal to the other packets.

Since a single modulated source is employed in the experiment, an optical coupler has been used to create two sequences of packets to be used as different inputs of the OAF. One sequence of packets that enters the SOA (via the MZI) on the right side (left propagating sequence of packets) is shown in Fig. 14(a). The other one sequence of packets that enters the SOA (via the optical circulator) on the left side (right propagating sequence of packets) is first delayed by $T_{p}$ as shown in Fig. 14(b). The time delay $T_{p}$ was set equal to one packet duration. This ensures that alternating conditions of packet contention between Packet 1 and Packet 2 take place. The coupler ratio was chosen to compensate for the different propagation losses experienced by the left and right propagating sequences of packets. The average optical power of the left propagating sequence of packets that entered the NPS via the MZI was $1.5 \mathrm{dBm}$ (measured before the MZI), while the average optical power of the right propagating sequence of packets that entered the NPS via the OC was $2.2 \mathrm{dBm}$ (measured before the $\mathrm{OC}$ ). The SOA was pumped with $126 \mathrm{~mA}$ of current.

The output of port 2 of the OAF is shown in Fig. 14(c). It is clearly visible that when contention takes place, no optical pulse is formed at the output of the OAF. However, an optical pulse is formed at output port 2 when no contention takes place. The extinction ratio between the average optical power of the optical pulse and the suppressed packet is $14.3 \mathrm{~dB}$, enough to drive the optical flip-flop memory [1], [5], [17].

\section{CONCLUSIONS}

We have demonstrated all-optical signal processing based on self-induced nonlinear polarization rotation in an SOA. Experimental and numerical results confirm that the mechanism behind the self-polarization rotation is the polarization dependent gain saturation as described in [15]. We have also demonstrated that a NPS based on SPR in a polarization switch interferometer
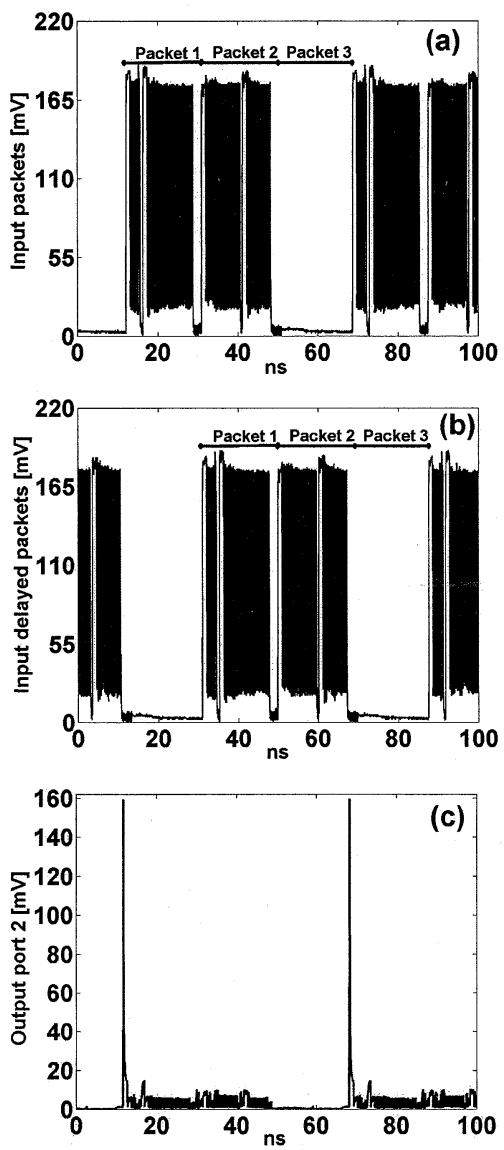

Fig. 14. Experimental results demonstrating the operation of the all-optical arbiter. (a) Optical input signal via the asymmetric Mach-Zehnder Interferometer (MZI); (b) Optical input signal via the Optical Circulator (OC); (c) Output of the all-optical arbiter (measured at the output port 2).

can be used to achieve optical signal processing functions that are useful for all-optical packet switching. It should be remarked that an NPS can only be utilized if the polarization of the input signal is well-defined. In system applications, this could be realized by employing a polarization independent regenerator in front of the NPS.

We have realized all-optical header processing function at a bit rate of $10 \mathrm{~Gb} / \mathrm{s}$, which can distinguish between two different header patterns by employing nonlinear polarization rotation 
in an SOA. We believe that by properly designing the header processor and the header patterns, a large number of different header patterns can be recognized. The header processor based on SPR has an extinction ratio between the header correlation pulse and the suppressed payload of $18.6 \mathrm{~dB}$, which is an improvement of $4.4 \mathrm{~dB}$ compared with results published in [1], [2], [17].

We have also demonstrated an $\mathrm{OAF}$ at a bit rate of $10 \mathrm{~Gb} / \mathrm{s}$. The arbiter can be used in a buffering system in a similar way as that presented in [17] and [21]. The main difference between our arbiter and the one presented in [17] and [21] is that the output of the arbiter presented in [17] and [21] was a CW signal. In this approach, the output of the arbiter is an optical pulse that drives the wavelength routing switch via an optical flip-flop [1], [5], [17].

We have also demonstrated a novel optical seed-pulse generator at a bit rate of $10 \mathrm{~Gb} / \mathrm{s}$ that produces a seed pulse synchronized with the beginning of the data packet. The seed pulse can be used to generate a local clock for packet synchronization. Moreover, the operation of the optical seed-pulse generator does not require any particular marker [6]-[9] in the packet format that identifies the beginning of the packet; rather, it uses the packet format itself. The extinction ratio between the seed pulse and the suppressed packet was $15 \mathrm{~dB}$. In addition, experimental results have shown that a seed pulse can be extracted even with an input signal extinction ratio of $5 \mathrm{~dB}$, confirming that the optical seed pulse generator could be used as the first stage in an optical cross-connect node.

Finally, experimental results show that the signal processing functions based on self-polarization rotation have a lower power operation and a higher extinction ratio than the same functions based on self-phase modulation.

\section{ACKNOWLEDGMENT}

The authors wish to thank R. Geldenhuys and A. K. Mishra and two anonymous referees for their comments on the manuscript.

\section{REFERENCES}

[1] M. T. Hill, A. Srivatsa, N. Calabretta, Y. Liu, H. de Waardt, G. D. Khoe, and H. J. S. Dorren, " $1 \times 2$ optical packet switch using all-optical header processing," Electron. Lett., vol. 37, pp. 774-775, 2001.

[2] N. Calabretta, Y. Liu, H. de Waardt, M. T. Hill, G. D. Khoe, and H. J. S. Dorren, "Multiple-output all-optical header processing technique based on two-pulse correlation principle," Electron. Lett., vol. 37, pp. 1238-1240, 2001

[3] N. Calabretta, Y. Liu, H. de Waardt, G. D. Khoe, and H. J. S. Dorren, "Bragg grating assisted all-optical header pre-processor," Electron. Lett., vol. 38, pp. 1560-1561, 2002.

[4] M. Zhao, J. de Merlier, G. Morthier, and R. Baets, "Experimental demonstration at $10 \mathrm{Gbps}$ of $2 \mathrm{R}$ optical regeneration in a fiber-based MZI with LOAs," in Proc. ECOC 2002, vol. 3, Copenhagen, Denmark, 2002, p. 7.3.6.

[5] M. T. Hill, H. de Waardt, G. D. Khoe, and H. J. S. Dorren, "All-optical flip-flop based on coupled laser diodes," IEEE J. Quantum Electron., vol. 37, pp. 405-413, Mar. 2001.

[6] M. C. Cardakli and A. E. Willner, "Synchronization of a network element for optical packet switching using optical correlators and wavelength shifting," IEEE Photon. Technol. Lett., vol. 14, pp. 1375-1377, Sept. 2002.
[7] T. J. Xia, Y. H. Kao, Y. Liang, J. W. Lou, K. H. Ahn, O. Boyraz, G. A. Nowak, A. A. Said, and M. N. Islam, "Novel self-synchronization scheme for high-speed packet TDM networks," IEEE Photon. Technol. Lett., vol. 11, pp. 269-271, Feb. 1999.

[8] D. Cotter, J. K. Lucek, M. Shabeer, K. Smith, D. C. Rogers, D. Nesset, and P. Gunning, "Self-routing of $100 \mathrm{Gbit} / \mathrm{s}$ packets using 6 bit 'keyword' address recognition," Electron. Lett., vol. 31, pp. 2201-2203, 1995.

[9] K.-L. Deng, I. Glesk, K. I. Kang, and P. R. Prucnal, "Unbalanced TOAD for optical data and clock separation in self-clocked transparent OTDM networks," IEEE Photon. Technol. Lett., vol. 9, pp. 830-832, June 1997.

[10] H. J. Lee, H. G. Kim, J. Y. Choi, and H. K. Lee, "All-optical clock recovery from NRZ data with simple NRZ-to-PRZ converter based on self-phase modulation of semiconductor optical amplifier," Electron. Lett., vol. 35, pp. 989-990, 1999.

[11] H. Soto, D. Erasme, and G. Guekos, "5-Gbit/s XOR optical gate based on cross-polarization modulation in semiconductor optical amplifier," IEEE Photon. Technol. Lett., vol. 13, pp. 335-337, Apr. 2001.

[12] R. J. Manning, A. Antonopoulos, R. Le Roux, and A. E. Kelly, "Experimental measurement of nonlinear polarization rotation in semiconductor optical amplifiers," Electron. Lett., vol. 37, pp. 229-231, 2001.

[13] Y. Liu, M. T. Hill, E. Tangdiongga, H. de Waardt, N. Calabretta, G. D. Khoe, and H. J. S. Dorren, "Wavelength conversion using nonlinear polarization rotation in a single semiconductor optical amplifier," IEEE Photon. Technol. Lett., vol. 15, pp. 90-92, Jan. 2003.

[14] Y. Liu, M. T. Hill, H. de Waardt, G. D. Khoe, D. Lenstra, and H. J. S. Dorren, "All-optical flip-flop memory based on two coupled polarization switches," Electron. Lett., vol. 38, pp. 904-906, 2002.

[15] H. J. S. Dorren, D. Lenstra, Y. Liu, M. T. Hill, and G. D. Khoe, "Nonlinear polarization rotation in semiconductor optical amplifiers: Theory and application to all-optical flip-flop memories," IEEE J. Quantum Electron., vol. 39, pp. 141-147, 2003.

[16] M. F. C. Stephens, M. Asghari, R. V. Penty, and I. H. White, "Demonstration of ultrafast all-optical wavelength conversion utilizing birefringence in semiconductor optical amplifiers," IEEE Photon. Technol. Lett., pp. 449-451, Apr. 1997.

[17] H. J. S. Dorren, M. T. Hill, Y. Liu, N. Calabretta, A. Srivatsa, F. M. Huijskens, H. de Waardt, and G. D. Khoe, "Optical packet switching and buffering by using all-optical signal processing methods," J. Lightwave Technol., vol. 21, Jan. 2003.

[18] F. Girardin, G. Guekos, and A. Houbavlis, "Gain recovery of bulk semiconductor optical amplifiers," IEEE Photon. Technol. Lett., vol. 10, pp. 784-786, June 1998.

[19] D. Cotter and A. D. Ellis, "Asynchronous digital optical regeneration and networks," J. Lightwave Technol., vol. 16, pp. 2068-2080, Dec. 1998.

[20] T. Sakamoto, A. Okada, M. Hirayama, Y. Sakai, O. Moriwaki, I. Ogawa, R. Sato, K. Noguchi, and M. Matsuoka, "Demonstration of an optical packet synchronizer for an optical packet switch," in Proc. OFC 2002, 2002, pp. 762-763.

[21] Y. Liu, M. T. Hill, N. Calabretta, H. de Waardt, G. D. Khoe, and H. J. S. Dorren, "All-optical buffering in all-optical packet switched crossconnects," IEEE Photon. Technol. Lett., vol. 14, pp. 849-851, June 2002.

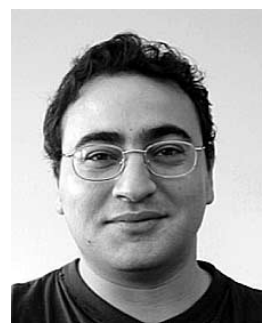

N. Calabretta was born on September 25, 1974 in Catanzaro, Italy. He received the Bachelor's degree and the M.S. degree, both in telecommunications engineering, from the Politecnico di Torino, Turin, Italy, in 1995 and 1999, respectively. He is currently working toward the Ph.D. degree with the COBRA Research Institute, the Eindhoven University of Technology, Eindhoven, The Netherlands.

In 1995, he visited the RAI Research Center (Italian broadcasting television), Turin, Italy, and developed an automatic procedure for designing and optimizing a 3-dB coupler at $2.5 \mathrm{GHz}$ in microstrip technology for an antenna system. At the Eindhoven University of Technology, he has investigated a novel cost-effective system for monitoring wavelength-division-multiplexing (WDM) channels. His field of interest is all-optical signal processing in a nonlinear medium, all-optical header recognition, all-optical synchronization, optical packet switching, and WDM monitoring system.

Mr. Calabretta is currently acting as a Referee for the IEEE PHOTONICS TECHNOLOGY LETTERS. In 1999, he won a scholarship for the European exchanging program Erasmus that enabled him to carry out his Master's thesis at the Faculty of Electrical Engineering, Eindhoven University of Technology. 


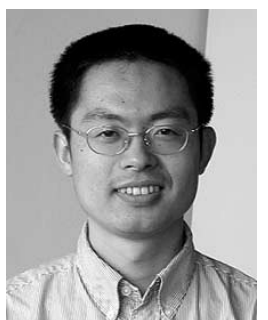

Y. Liu (S'02) was born in Sichuan province, China, in 1970. He received the M.S. degree in electronic engineering from the University of Electronic Science and Technology of China, Sichuan, in 1994. He is currently working toward the Ph.D. degree with the Eindhoven University of Technology, Eindhoven, The Netherlands.

From 1994 to April 2000, he taught and conducted research at the University of Electronic Science and Technology of China. In April 2000, he came to the Eindhoven University of Technology. His field of interest is all-optical buffering by using all-optical signal processing.

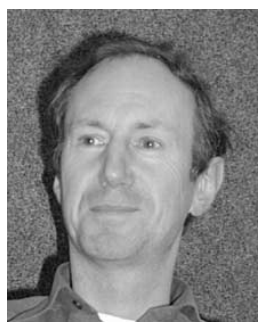

F. M. Huijskens was born in Oudenbosch, The Netherlands, in 1958. He graduated in applied physics from the Technical College of Dordrecht, Dordrecht, The Netherlands, in 1979.

From 1981 to 1984, he was an Electronic Test Engineer at Siemens Gammasonics. In 1985, he joined the Electro-Optical Communications Group of Eindhoven University of Technology, Eindhoven, The Netherlands. His work has involved research on passive fiber couplers and support on projects concerning phase and polarization diversity coheren systems. He has contributed to the development of optical cross-connect demonstrators and in packaging technologies of optical integrated devices. Recently, he has assisted in fields of optical packet switching and PDFAs.

M. T. Hill (M'96-A'97), photograph and biography not available at the time of publication.

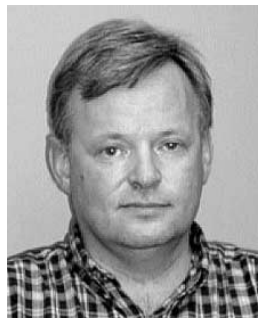

H. de Waardt was born in Voorburg, The Netherlands, on December 1, 1953. He received the M.Sc. and $\mathrm{Ph} . \mathrm{D}$. degrees in electrical engineering from the Delft University of Technology, Delft, The Netherlands, in 1980 and 1995, respectively.

In 1981, he joined the Department of Physics, KPN Research, Leidschendam, where he was engaged in research on the performance aspects of long-wavelength semiconductor laser diodes, LEDs, and photodiodes. In 1989, he moved to the Department of Transmission, where he has been working in the fields of high-bit-rate direct-detection systems, optical preamplification, wavelength-division multiplexing (WDM), dispersion-related system limitations, and the system application of resonant optical amplifiers. He contributed to national and international standardization bodies and to the EURO-COST activities 215 and 239. In October 1995, he was appointed Associate Professor with the Faculty of Electrical Engineering in the area of high-speed trunk transmission at the University of Eindhoven, Eindhoven, The Netherlands. He was active in such European research programs as ACTS BLISS, ACTS Upgrade, and ACTS APEX. At present, he coordinates the TU/e activities in the European projects IST METEOR and IST FASHION. He is Member of the project management committee of the national project BTS RETINA. He has authored or coauthored more than 60 refereed papers and conference contributions. His current research interests are in applications of semiconductor optical amplifiers, high-speed optically time-division-multiplexed (OTDM) transmission, and WDM optical networking.

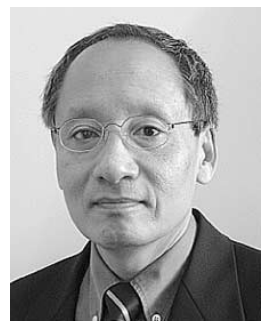

G. D. Khoe (S'71-M'71-SM'85-F'91) was born in Magelang, Indonesia, on July 22, 1946. He received the Elektrotechnisch Ingenieur degree (cum laude) from the Eindhoven University of Technology, Eindhoven, The Netherlands, in 1971.

He started research at the Dutch Foundation for Fundamental Research on Matter (FOM) Laboratory on Plasma Physics, "Rijnhuizen," Neuwegein, The Netherlands. In 1973, he moved to the Philips Research Laboratories to start research in the area of optical fiber communication systems. In 1983, he was appointed Part-Time Professor at the Eindhoven University of Technology. He became a Full Professor at the same university in 1994 and is currently Chairman of the Department of Telecommunication Technology and Electromagnetics (TTE). Most of his work has been devoted to single-mode-fiber systems and components. Currently his research programs focus on ultrafast all-optical signal processing, high-capacity transport systems, and systems in the environment of the users. He has more than 40 U.S. patents and has authored and coauthored more than 100 papers, invited papers, and book chapters. He has been involved in journal activities as Associate Editor, Member of the Advisory Board, or Reviewer. Today, he is an Associate Editor of $J Q E$. In Europe, he is closely involved in research programs of the European community and in Dutch national research programs, as participant, evaluator, auditor, and program committee member. $\mathrm{He}$ is one of the founders of the Dutch COBRA University Research Institute.

Dr. Khoe is Junior Past President of the IEEE Lasers \& Electro-Optics Society (LEOS). His has served in the IEEE LEOS organization as European Representative in the BoG, VP Finance \& Administration, BoG Elected Member, and Member of the Executive Committee of the IEEE Benelux Section. He was also founder of the LEOS Benelux Chapter. Among his many professional activities, he has served as Member or Chairman on technical, management, and advisory committees for many conferences, and he recently served as General Co-Chair of the ECOC 2001. In 2001, he brought four groups together to start a new international alliance called the European Institute on Telecommunication Technologies (eiTT). He was one of the three recipients of the prestigious Top Research Institute Photonics grant that was awarded to the Dutch COBRA University Research Institute in 1998 by the Netherlands Ministry of Education, Culture and Science and received the MOC/GRIN award in 1997.

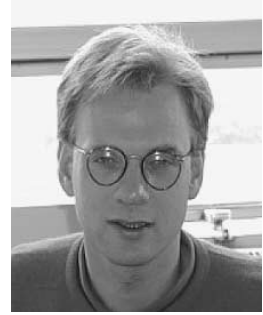

H. J. S. Dorren received the M.Sc. degree in theoretical physics and the Ph.D. degree from Utrecht University, Utrecht, The Netherlands, in 1991 and 1995, respectively.

After a postdoctoral position at Utrecht University, he joined the Eindhoven University of Technology, Eindhoven, The Netherlands, in 1996, where he presently serves as an Associate Professor. In 2002, he was also a Visiting Researcher at the National Institute of Industrial Science and Technology (AIST), Tsukuba, Japan. His research interest include optical packet switching, digital optical signal processing, and ultrafast photonics.

Dr. Dorren received a VIDI award from the Netherlands Organization for Scientific Research in 2002. 